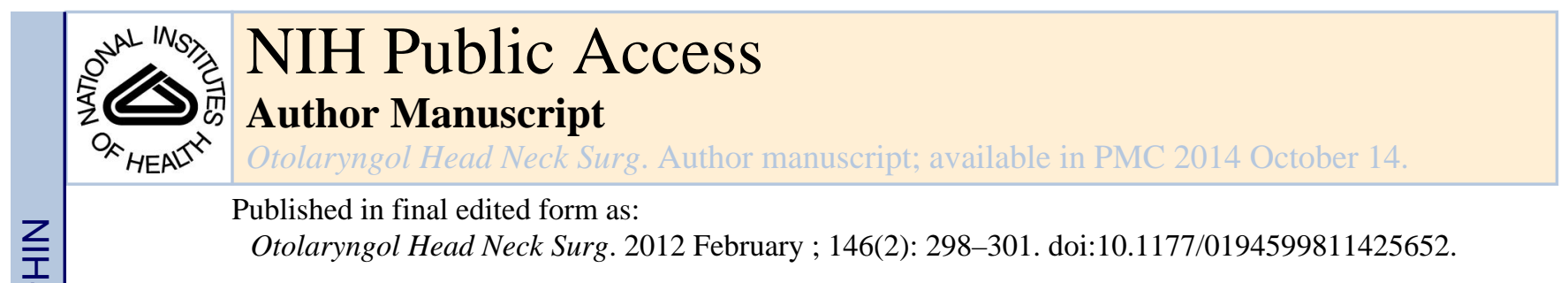

\title{
Risk Factors for Supraglottoplasty Failure
}

\author{
Kristine E. Day ${ }^{1}$, Christopher M. Discolo, MD¹, Jeremy D. Meier, MD¹, Bethany J. Wolf, \\ PhD², Lucinda A. Halstead, MD1, and David R. White, MD1 \\ ${ }^{1}$ Department of Otolaryngology-Head and Neck Surgery, Medical University of South Carolina, \\ Charleston, South Carolina, USA \\ ${ }^{2}$ Department of Medicine, Division of Biostatistics and Epidemiology, Medical University of South \\ Carolina, Charleston, South Carolina, USA
}

\begin{abstract}
Objective-To review outcomes after supraglottoplasty for laryngomalacia and identify risk factors for supraglottoplasty failure.
\end{abstract}

Study Design-Case series with chart review.

Setting-Tertiary care children's hospital.

Subjects and Methods-Retrospective case series evaluating patient outcomes after supraglottoplasty at an academic medical center between 2004 and 2010. Surgical failure was defined as need for revision surgery, tracheostomy tube placement, or gastrostomy tube insertion. Multivariable logistic regression was performed to identify risk factors for failure.

Results-The authors identified 95 children who underwent supraglottoplasty. After excluding patients with inadequate follow-up data, 74 patients were included. On the basis of chart review, $12(16 \%)$ of those patients were defined as failures according to the criteria above. Age, history of prematurity ( $<34$ weeks' gestational age), weight, growth curve percentile, neurologic/ developmental problems, genetic syndrome, cardiac abnormality, synchronous airway lesions, and surgical technique were considered in risk factor analysis. Multivariable logistic regression was performed, revealing history of prematurity to be the only independent risk factor for failure (odds ratio $=4.85 ; 95 \%$ confidence interval, $1.07-22.1 ; \mathrm{P}=.041)$.

Conclusions-Outcomes after supraglottoplasty were comparable to previous reports in the literature. History of prematurity should be considered a risk factor for surgical failure.

\section{Author Contributions}

Kristine E. Day, conception and design, collection, analysis and interpretation of data, drafting and revising the article, final approval of the manuscript; Christopher M. Discolo, conception and design, analysis and interpretation of data, drafting and revising the article, final approval of the manuscript; Jeremy D. Meier, conception and design, collection, analysis and interpretation of data, drafting and revising the article, final approval of the manuscript; Bethany J. Wolf, conception and design, analysis and interpretation of data, drafting and revising the article, final approval of the manuscript; Lucinda A. Halstead, analysis and interpretation of data, revising the article, final approval of the manuscript; David R. White, conception and design, collection, analysis and interpretation of data, drafting and revising the article, final approval of the manuscript.

Disclosures

Competing interests: David R. White is a consultant for Gyrus and Medtronic. Christopher M. Discolo received a grant from Otosonics, Inc, to study otitis media.

Sponsorships: None. 


\section{Introduction}

Laryngomalacia is the most common congenital anomaly of the larynx and cause of stridor in newborn children. ${ }^{1-3}$ Upper airway obstruction occurs due to supraglottic collapse during inspiration. ${ }^{1}$ Although the exact pathophysiology is unknown, the tissues involved include the aryepiglottic folds, arytenoid mucosa, and the epiglottis. ${ }^{1}$ The diagnosis is usually made by flexible fiber-optic laryngoscopy, which demonstrates shortening of the aryepiglottic folds and/or redundant arytenoid mucosa, with or without epiglottic prolapse. ${ }^{3,4}$ Most cases are present at birth, reach a peak in severity at around 6 months of age, and resolve without intervention during the second year of life. ${ }^{1,2}$ In $10 \%$ to $20 \%$ of patients, however, laryngomalacia will become severe enough to warrant surgical intervention..$^{2-4}$ Failure to thrive, feeding difficulties, upper airway obstruction, and severe dyspnea are some of the common indications for surgery.

Supraglottoplasty has become the mainstay of surgical management for severe laryngomalacia. The procedure typically involves division of the aryepiglottic folds and resection of supraglottic tissue. Success rates of $38 \%$ to $100 \%$ have been reported with relatively low complication rates. ${ }^{2} \mathrm{O}^{\prime}$ Donnell et $\mathrm{al}^{5}$ achieved a $90 \%$ success rate, defined by improvement in stridor, and describe the procedure as low risk. Despite reported high success rates, $19 \%$ to $45 \%$ of children will require a revision of the original procedure or insertion of a tracheotomy tube to bypass persistent obstruction. ${ }^{2}$ A study by Denoyelle et al1 found that the presence of associated congenital anomalies is a risk factor for surgical failure. Indeed, patients with isolated laryngomalacia fare much better in terms of discharge dates, rates of pneumonia, unplanned pediatric intensive care unit (PICU) admissions, and symptomatic control at follow-up than patients with significant comorbidities. ${ }^{5}$ In 2009 , Schroeder et al6 found that patients with neurological conditions, mandibular hypoplasia, subglottic stenosis greater than $35 \%$, or preexisting laryngeal edema were more likely to have a complicated postoperative course. Hoff et $\mathrm{al}^{2}$ later substantiated that the number and type of medical diagnoses a patient carries directly affect whether supraglottoplasty will succeed. In particular, patients with neurologic and cardiac comorbidities seem to carry a higher rate of supraglottoplasty failure. Age may also play a factor as this study also showed that patients younger than 2 months of age without comorbidities had a higher rate of revision.

The purpose of this study is to review our patient outcomes after supraglottoplasty and identify risk factors associated with treatment failure in our series. Identifying factors that increase the likelihood for a poor outcome may help to better define treatment algorithms for laryngomalacia.

\section{Methods}

This study is a retrospective case series evaluating patient outcomes after supraglottoplasty at the Medical University of South Carolina (MUSC, Charleston, South Carolina) between 2004 and 2010. MUSC Institutional Review Board for Human Research approval was obtained prior to the study. A total of 95 children underwent supraglottoplasty for the 
diagnosis of laryngomalacia. After exclusion of patients with inadequate follow-up data, 74 patients, aged 1 day to 7.9 years, were included in the study.

Patients were diagnosed with laryngomalacia requiring surgical intervention based on clinical presentation and confirmed by conscious flexible fiber-optic laryngoscopy either in the office setting or via direct visualization in the operating room prior to surgery. Patients are routinely treated for gastroesophageal reflux disease (GERD) in the perioperative period using a combination of histamine (H2) receptor antagonists and/or proton pump inhibitors (PPIs). Supraglottoplasty was performed using cold steel (CS) laryngeal microinstruments or the $\mathrm{CO} 2$ laser. The larynx was suspended and the operation performed under binocular microscopic visualization. Most of the operations included excision of the redundant tissue overlying the arytenoid cartilage. In all cases, the aryepiglottic folds were incised.

Patient charts were examined for the following: age, history of prematurity ( $<34$ weeks' gestational age), weight at the time of surgery, growth curve percentile, neurologic/ developmental problems, genetic syndrome, cardiac abnormality, synchronous airway lesions, and surgical technique. Synchronous airway lesions included subglottic stenosis and tracheomalacia. Surgical failure was defined as need for postoperative revision surgery, tracheotomy tube, or gastrostomy tube.

\section{Statistical Analysis}

The main outcome of interest in these data was success or failure for the operation. Failure was defined as a patient requiring 1 or more of the following postsurgery: (1) revision operation, (2) tracheotomy, or (3) gastrostomy tube. Explanatory variables in the data included age in days, history of prematurity ( $<34$ weeks' gestational age), weight in kilograms, weight percentile, neurologic or developmental problems (yes/no), genetic syndrome (yes/no), cardiac abnormality (yes/no), synchronous airway lesions (yes/no), and type of surgery (CS vs laser). Weight at time of surgery was log transformed for all statistical analyses.

Prior to modeling surgical success, descriptive statistics were calculated for all variables using SAS version 9.2. ${ }^{7}$ We performed Fisher exact tests on all dichotomous variables and independent Student $t$ tests for continuous variables to examine the association between surgical success and all explanatory variables. Explanatory variables with a P value of $<.20$ were considered in a multivariable logistic regression of probability of surgical success model. ${ }^{8}$ The final logistic regression model was selected using backwards selection. All $\mathrm{P}$ values reported in the multivariate model were 2-sided, and the type I error rate was set at 0.05. All statistical analyses were performed using SAS version 9.2. ${ }^{7}$

\section{Results}

The study population included 74 subjects of whom $66.2 \%$ were male. The median age at the time of surgery was 116.5 days, and the median weight at the time of the surgery was 5.5 $\mathrm{kg}$, with half of the participants being at or under the 30th percentile for weight based on age. We report the results for all health-related variables in Table 1 . Nearly half of the 
patients had 1 or more additional airway lesions. The most common synchronous airway lesions included subglottic stenosis (15\%) and tracheomalacia (9\%).

In the bivariate analysis of surgical success with each explanatory variable (Table 1), only prematurity was found to be significantly associated with surgical success $(\mathrm{P}=.05)$. A nonsignificant trend of surgical success was noted when considering log of weight at time of surgery and growth percentile $(\mathrm{P}=.11$ and $\mathrm{P}=.13$, respectively). We considered prematurity, the log-transformed weight at time of surgery, and growth percentile in a multivariable logistic regression model. We also controlled for type of surgery and age at time of surgery in the full model. However, only prematurity was still significantly associated with surgical failure in the multivariable logistic regression model. Thus, the final multivariable logistic regression model included prematurity and controlled for age at time of surgery and type of surgery (Table 2). The odds of surgical failure among children who were born prematurely were 4.85 times the odds of surgical failure among children not born prematurely, controlling for age at time of surgery and type of surgery (95\% confidence interval [CI], 1.07-22.1; P = .04).

\section{Discussion}

Congenital anomalies and medical comorbidities have been found to adversely affect supraglottoplasty outcomes. Denoyelle et $\mathrm{al}^{1}$ found a significantly increased risk of failure or only partial improvement in patients with associated congenital anomalies such as Pierre Robin sequence, psychomotor retardation, encephalopathy, CHARGE syndrome, or Down syndrome. Additional studies have identified age less than 2 months, ${ }^{2}$ cardiac comorbidities, ${ }^{2}$ and neurological impairment ${ }^{6,9}$ to increase the risk of poor surgical outcome. Synchronous airway lesions such as subglottic stenosis or preexisting laryngeal edema have also adversely affected the postoperative course. ${ }^{6}$ In that series, subglottic stenosis and preexisting laryngeal edema were independent risk factors requiring increased airway support after supraglottoplasty. Our series did not reveal synchronous airway lesions as an independent risk factor for poor surgical outcomes as defined by our criteria. These differences in outcome measures highlight some of the challenges encountered in the literature when reviewing laryngomalacia treatment results.

In the series discussing synchronous airway lesions, prematurity was not found to be an independent risk factor for a poor outcome after supraglottoplasty. However, a premature birth was found at a much higher rate in the patients with neurological conditions. Our series identifies prematurity as an independent risk factor for a poor outcome after supraglottoplasty. A history of prematurity remained significant even after controlling for a subject's age and weight. Because of the retrospective design, we are unable to determine the reason for these poorer outcomes in children with a history of prematurity.

A number of factors are believed to play a role in the development of laryngomalacia, but the precise pathophysiology is not completely understood. GERD is frequently implicated in the etiology of laryngomalacia. ${ }^{10}$ All of our patients are treated perioperatively with antireflux medications because it is believed that GERD may cause and/or exacerbate laryngomalacia. Structural anatomic abnormalities have also been suggested as a cause for 
laryngomalacia. An omega-shaped epiglottis, shortened aryepiglottic folds, and redundant supra-arytenoid tissue are frequently seen. Additional theories attribute the problem to abnormally pliable or immature cartilage. ${ }^{11}$ Recently, Thompson ${ }^{11}$ proposed that abnormal sensorimotor integrative function of the larynx may play a central role in the etiology of laryngomalacia. Bluestone ${ }^{12}$ suggests that evolution has forced human gestation to be 12 months shorter than needed. He proposes that many pediatric otolaryngologic conditions, including laryngomalacia and otitis media, are encountered because of humans' gestational length. Although we cannot draw conclusions from this study to support these theories, perhaps in utero maturation of laryngeal cartilage and neural pathways is underestimated.

Unlike other studies, our series did not reveal adverse outcomes in patients with other neurologic, cardiac, or synchronous airway lesions. The low failure rate combined with the small number of patients within each subgroup with these comorbidities limits our ability to identify significance. Increasing our overall sample size and the number of patients with comorbidities would improve our ability to identify other potential adverse factors. Also, varying definitions of a poor surgical outcome between studies may also contribute to these discrepancies.

Limitations of this study include the retrospective method of data collection. A significant number of patients could not be included because follow-up data were not available. Although we cannot assume the results of those lost to follow-up, it is possible that the failure rate represented in this report is higher than the actual results as we predict that many of those patients had unavailable postoperative data because they significantly improved after intervention and sought follow-up care in their local community. Also, the small number of patients with poor surgical outcome (only 16\%) resulted in limited statistical power, which may explain why some variables were not shown to be significantly associated with surgical outcome. A larger sample size would be required to determine, for instance, if being premature at birth and weight at the time of surgery (which were significantly associated) were independently associated with surgical outcome. Another challenge encountered when evaluating supraglottoplasty outcomes is the lack of a universal definition of success or failure. Most series use subjective outcomes such as improvement in stridor or resolution of feeding difficulties to measure success. Objective measures such as those used in this series (revision supraglottoplasty, tracheotomy, gastrostomy tube) have been evaluated, but these are often limited by low failure rates and small case series. The large population of supraglottoplasty patients at our institution reduced the impact of these limitations. Additional objective assessments of supraglottoplasty outcomes such as polysomnography ${ }^{13}$ or growth velocity ${ }^{14}$ were not practical given the design of this study.

\section{Conclusions}

Outcomes after supraglottoplasty in our series were comparable to previous reports in the literature. The findings from this multivariable analysis suggest that a history of prematurity should be considered a risk factor for surgical failure. The cause of poorer outcomes after supraglottoplasty in patients with a history of prematurity is not known. As the pathophysiology of laryngomalacia and the link to the immature larynx are investigated, the impact of prematurity may be better elucidated. 


\section{Acknowledgments}

Funding source: South Carolina Clinical \& Translational Research Institute, Medical University of South Carolina's CTSA, National Institutes of Health (NIH)/National Center for Research Resources (NCRR) grant number UL1RR029882. The contents are solely the responsibility of the authors and do not necessarily represent the official views of the NIH or NCRR.

\section{References}

1. Denoyelle F, Mondain M, Grésillon N, et al. Failures and complications of supraglottoplasty in children. Arch Otolaryngol Head Neck Surg. 2003; 129:1077-1080. [PubMed: 14568790]

2. Hoff S, Schroeder J Jr, Rastatter J, Hollinger L. Supraglottoplasty outcomes in relation to age and comorbid conditions. Int J Pediatr Otorhinolaryngol. 2010; 74:245-249. [PubMed: 20022388]

3. Thompson D. Laryngomalacia: factors that influence disease severity and outcomes of management. Curr Opin Otolaryngol Head Neck Surg. 2010; 18:564-570. [PubMed: 20962644]

4. Avelino M, Liriano R, Fujita R, et al. Management of laryngomalacia: experience with 22 cases. Braz J Otorhinolaryngol. 2005; 71:330-334. [PubMed: 16446937]

5. O'Donnell S, Murphy J, Bew S, Knight LC. Aryepiglottoplasty for laryngomalacia: results and recommendations following a case series of 84. Int J Pediatr Otorhinolaryngol. 2007; 71:12711275. [PubMed: 17597233]

6. Schroeder JW, Bhandarkar ND, Holinger LD. Synchronous airway lesions and outcomes in children with severe laryngomalacia requiring supraglottoplasty. Arch Otolaryngol Head Neck Surg. 2009; 135:647-651. [PubMed: 19620584]

7. SAS Institute, Inc.. SAS System for Windows, version 9.2. SAS Institute, Inc; Cary, NC: 2009.

8. Mickey J, Greenland S. A study of the impact of confounder-selection criteria on effect estimation. Am J Epidemiol. 1989; 129:125-137. [PubMed: 2910056]

9. Fraga JC, Schopf L, Volker V, Canani S. Endoscopic supraglottoplasty in children with severe laryngomalacia with and without neurological impairment. J Pediatr. 2001; 77:420-424.

10. Giannoni C, Sulek M, Friedman EM, Duncan NO III. Gastroesophageal reflux association with laryngomalacia: a prospective study. Int J Pediatr Otorhinolaryngol. 1998; 43:11-20. [PubMed: 9596365]

11. Thompson DM. Abnormal sensorimotor integrative function of the larynx in congenital laryngomalacia: a new theory of etiology. Laryngoscope. 2007; 117:1-33. [PubMed: 17513991]

12. Bluestone CD. Humans are born too soon: impact on pediatric otolaryngology. Int J Pediatr Otorhinolaryngol. 2005; 69:1-8. [PubMed: 15627440]

13. O'Connor TE, Bumbak P, Vijayasekaran S. Objective assessment of supraglottoplasty outcomes using polysomnography. Int J Pediatr Otorhinolaryngol. 2009; 73:1211-1216. [PubMed: 19524306]

14. Whymark MD, Clement A, Kubba H, Geddes N. Laser epiglottopexy for laryngomalacia: 10 years' experience in the west of Scotland. Arch Otolaryngol Head Neck Surg. 2006; 132:978-982. [PubMed: 16982974] 
